\title{
Microcavity-Enhanced Fluorescence Energy Transfer from Quantum Dot-Excited Whispering Gallery Modes to Acceptor Dye Nanoparticles
}

\author{
Subha Jana, ${ }^{1}$ Xiangzhen $\mathrm{Xu},{ }^{1}$ Andrey Klymchenko, ${ }^{2}$ Andreas Reisch, ${ }^{2}$ Thomas Pons ${ }^{1, *}$ \\ 1 Laboratoire de Physique et d'Étude des matériaux (LPEM, UMR 8213), ESPCI Paris, \\ Université PSL, CNRS, Sorbonne Université, 75005 Paris, France
}

2 Université de Strasbourg, CNRS, Laboratoire de Bioimagerie et Pathologies UMR 7021, F67000 Strasbourg, France

Corresponding author: thomas.pons@espci.fr

\begin{abstract}
Whispering gallery mode (WGM) microcavities are emerging as potential candidates in the field of biosensing applications, as their resonance wavelengths shift with changes in the refractive index in the region of their evanescent field. Their high-quality resonance modes and accessible surface functionalities make them promising for molecular assays but their high sensitivity makes them inherently unstable. Here we demonstrate that WGM resonances also strongly enhance fluorescence energy transfer between donors placed inside the microcavity and acceptors placed outside. We load colloidal quantum dots (QDs) into polymeric microspheres, to provide WGMs that benefit from the QD optical features when used as energy transfer donors. Spectroscopic analysis of the emission from the microcavities shows that the high quality of WGMs enables a very efficient energy transfer to dyeloaded polymer nanoparticle (dyeNP) acceptors placed in their vicinity. Compared to Förster Resonance Energy Transfer, WGM-enabled energy transfer (WGET) occurs over a much more extended volume, thanks to the delocalization of the mode over a typically $10^{5}$ times larger surface and to the extension of the WGM electromagnetic field to larger distances (> $100 \mathrm{~nm}$ vs. a few $\mathrm{nm}$ ) from the surface of the microcavity. The resulting sensing scheme combines the sensitivity of WGM spectroscopy with the specificity and simple detection schemes of fluorescence energy transfer, thus providing a potentially powerful class of biosensors.
\end{abstract}

\section{Keywords:}

energy transfer, microcavity, whispering gallery mode, quantum dot, nanoparticle 
Förster resonance energy transfer (FRET) is a photophysical process that is broadly used as the underlying principle of many bio-detection schemes. ${ }^{1-3}$ FRET is defined as the transfer of electronic excitation from one quantum emitter (donor) to another (acceptor) via a radiation-less dipole-dipole interaction. The FRET rate is inversely proportional to the sixth power of the separation distance between the donor and the acceptor; this sharp dependence is used to detect assemblies of biomolecules labeled respectively by donor and acceptor entities, or to probe changes in biomolecular conformations in the 3-10 nm range. As FRET directly translates into simple, easily detectable, fluorescence outputs such as donor/acceptor intensities or lifetimes, it has become a ubiquitous tool to probe various biological and chemical events such as protein-protein, ${ }^{4,5}$ DNA-protein, ${ }^{6,7}$ RNAprotein $^{8}$ interactions, protein conformational changes, ${ }^{9,10}$ detection of ions, ${ }^{11,12}$ drugs $^{13,14}$ and other biological moieties. ${ }^{15,16}$ However, for larger biological entities and donor-acceptor separation distances, the rate of FRET can be substantially low, making the detection technique inefficient and inconclusive.

In the last few years, different schemes have been developed to increase the distance range of energy transfer, using for example energy migration in nano-assemblies, ${ }^{17,18}$ energy transfer to metallic nanoparticles ${ }^{19-21}$ or between lanthanides and semiconductor quantum dots (QDs)..$^{22,23}$ Optical microcavities have emerged as a potential candidate for bio-sensing schemes owing to their advantages such as improved sensitivity, ease of fabrication and the prospect of use in homogenous assays. Various kinds of optical microcavities have been explored like Fabry-Perot cavities, ${ }^{24,25}$ whispering gallery modes (WGMs) from dielectric microspheres, ${ }^{26,27}$ photonic crystals ${ }^{28,29}$ and ring resonators. ${ }^{30,31}$ In particular, the success of microsphere WGM sensors relies on the extension of the mode as an evanescent field within a few tens to hundreds of $\mathrm{nm}$ above the microsphere surface. Changes in the refractive index within this WGM evanescent field (e.g. due to binding of biomolecules, viruses...) leads to a shift in the WGM wavelength, which can be very accurately determined thanks to the high quality factor ( $Q$ factor) of polymer or silica microsphere WGMs $\left(10^{4}-10^{6}\right)$. However, this extreme sensitivity is also a source of instability since fluctuations in temperature, solution refractive index and nonspecific binding of biomolecules also cause WGM shift over time. There is therefore a strong interest in combining the sensitivity and distance range of WGM sensors with the specificity provided by energy transfer assays. Many studies have revealed that the efficiency of energy transfer can be enhanced by several orders of magnitude when the donor and acceptor moieties are present inside the same cavity at both small $(<\lambda / 10)$ or large $(>\lambda / 10)$ separations. In micro-cavities of relatively low $\mathrm{Q}$-factor, the non-radiative FRET rate varies linearly with the donor emission rate, which is accelerated due to the modified photonic mode density inside the cavity. ${ }^{32}$ In addition, micro-cavities 
with high Q-factor can mediate long-range radiative energy transfer between the donor and acceptor. This enhancement due to the coupling between the dipoles of the emitters and the morphology dependent WGMs of the microcavity is explained with a simple model by Druger et al..$^{33}$ In a recent study, Kushida et al. reported long range propagation of light from WGMs between different microspheres, and energy transfer to acceptor species within microspheres doped with both donor and acceptor chromophores. ${ }^{34}$ In these studies, energy transfer occurs between donor and acceptor species located within the same microsphere at high concentration. In these configurations, energy transfer is not very sensitive to environmental conditions since the concentration and location of both fluorescent species are fixed. Two other studies reported the energy transfer between optical donors placed in the vicinity of microsphere to modes or to acceptors placed inside the microsphere. ${ }^{35,36}$

In our study, only donors are embedded within the microcavity, and acceptors come from the outside within its evanescent field. This configuration makes it possible, for example, to design assays in which the acceptors bind to the surface of the cavity when a biomolecular target is present, similarly to FRET sandwich assays. We report the fabrication and study of polystyrene-based microcavities with high $(>4,000)$ Q-factor that are doped with colloidal quantum dots (QDs) and can act as an optical donor, enabling us to perform energy transfer in longer range than the traditional FRET limit. The use of QDs not only makes the micro-cavities bright and photostable but also provides us the flexibility to tune the WGM wavelengths throughout the whole visible spectrum. The QDs also have very high absorbance in the UV region $(<400 \mathrm{~nm})$ where the absorption from the acceptors is negligible. This provides us with a broad range of wavelengths to excite the donor QDs without exciting the acceptors directly, greatly simplifying the detection of energy transfer processes. ${ }^{23,37}$ As FRET acceptors we used polymer nanoparticles loaded with the salt of a rhodamine $B$ derivative and a bulky hydrophobic counterion (dyeNPs), ${ }^{38}$ which were assembled on the surface of the micro-cavities to demonstrate whispering gallery mode-mediated energy transfer (WGET, see Scheme 1). We analyzed the donor and acceptor fluorescence intensities and changes in the quality factor of WGMs to estimate the dependence of the WGET rate on the overlap with the acceptor absorption spectrum and on the acceptor surface density. We examined the impact of the high Qfactor on energy transfer efficiency from the WGMs to optical acceptors present within its evanescent field. Finally, we compare the energy transfer efficiency between WGET and standard FRET in the context of biosensing, as a function of acceptor surface density and separation distances. 


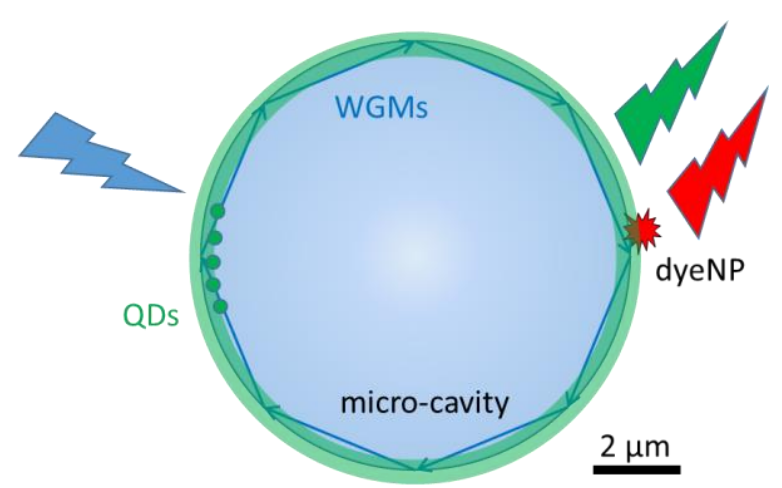

Scheme 1. Whispering gallery mode energy transfer (WGET) from QDs coupled to WGMs to dyeNPs placed closed to the surface, within the evanescent field of the micro-cavity.

\section{Results and discussion:}

Two batches of core-shell semiconductor quantum dots were synthesized using solvothermal methods with emission spectra centered respectively at $505 \mathrm{~nm}$ and $525 \mathrm{~nm}$ (Figure 1 and Figure S1). They are hereafter referred to as QD505 and QD525, respectively. This enables tuning the overlap of their emission with the absorption spectrum of the acceptor. We incorporate the QDs into commercial polystyrene microparticles using infusion in a chloroform-butanol mixture (Figure $2 \mathrm{~A}$ ). The chloroform causes swelling of the polystyrene matrix and opens nanopores into which QDs may diffuse from the outer solution phase. The microspheres are then separated from excess QDs and resuspended into water.



Figure 1: (From left to right respectively) Absorption spectrum of QD 525 (dotted), emission spectra of QD 505 (black) and QD 525 (blue), absorption (green, dashed) and emission (purple, dashed) spectra of the acceptor dye NPs 
When observed in a confocal fluorescence microscope, the beads show a bright fluorescent ring (Figure $2 \mathrm{~B}$ ), the thickness of which is limited by the resolution of the microscope, ca. $0.5 \mu \mathrm{m}$. This suggests that the QDs have only penetrated a few hundreds of $n m$ deep into the outer layer of the microspheres, as previously reported in similar systems. ${ }^{39}$

The emission spectrum of the collected fluorescence is shown in Figure $2 \mathrm{C}$, where the confocal volume has been placed at the equator of the microsphere loaded with QD525. The spectrum shows a broad envelope emission, corresponding to the spectrum of QD emission in free space. In addition, the spectrum shows a series of regularly spaced pairs of peaks, corresponding to coupling of the QD emission to the transverse magnetic (TM) and electric (TE) whispering gallery modes of the microspheres. In comparison, when the detection pinhole is located away from the excitation spot, the emission spectrum consists mainly in the WGM peaks with minimal contribution from the free space emission (Figure S2). Finally, emission spectra collected from whole beads in a wide-field microscopy setup also show similar features as in Figure $2 \mathrm{C}$ (see Figure S3). According to Lam et $a \mathrm{l}^{40}$ the wavelengths of WGMs of a microsphere of radius $R$ can be expressed asymptotically as a function of the angular mode number $\mathrm{I}$, radial mode number $i$, using the dimensionless parameter $x=2 \pi R / \lambda$ :

$$
x \sim v+a_{i}\left(\frac{v}{2}\right)^{1 / 3}-\frac{m p}{\sqrt{m^{2}-1}}+0.3 a_{i}{ }^{2}(4 v)^{-1 / 3}+\frac{m^{3} p\left(2 p^{2} / 3-1\right) a_{i}}{\left(2 v^{2}\right)^{1 / 3}\left(m^{2}-1\right)^{3 / 2}}
$$

where $=l+\frac{1}{2},=n_{S} / n_{e}$, where $n_{s}$ and $n_{e}$ are the refractive indices of the microspheres and the environment solution, $p=1$ for TE and $p=1 / \mathrm{m}^{2}$ for TM modes, and $a_{i}$ is the $\mathrm{i}^{\text {th }}$ zero of the Airy function. Here we only consider $\mathrm{i}=1$ modes, since the widths of higher order modes are much higher and are not distinguishable in our spectra. Assuming a refractive index of 1.33 for the water solution, the series of peaks can be fitted with a unique set of $R$ and $n_{s}$ parameters. ${ }^{41}$ For the spectrum shown in Figure 2C, for angular mode numbers ranging from $475 \mathrm{~nm}$ to $575 \mathrm{~nm}$, this corresponds to a radius of 3.82 $\mu \mathrm{m}$ and a refractive index of 1.798 (see Figure S4). In general, all the fitted radii lie within the 3.5-6 $\mu \mathrm{m}$ range of the distribution of the microspheres. The fitted microsphere refractive indices are all above the refractive index of polystyrene $\left(n_{P S}=1.59-1.6\right),{ }^{42}$ which can be attributed to the contribution of the QDs to the effective refractive index of the microsphere outer layer. When acquired with a higher resolution spectrofluorimeter, the widths of the emission peaks are measured as typically 0.125 $\mathrm{nm}$ (Figure S5). This corresponds to the spectrometer resolution limit. This shows that we are not able to resolve the mode spectral width with this technique, and that the quality factor of the modes, $Q=$ $\lambda / \Delta \lambda$, is greater than 4,200 . This value is only a lower bound, and previous studies showed that WGM quality factors in similar polymer microspheres can exceed $10^{6}$ in water. ${ }^{43}$ The wavelength range of the excited WGMs can be easily tuned by choosing the appropriate QD population (Figure S6). In 
addition, the good photo-stability of QDs enable repeated acquisition with minimal photo-bleaching over time (Figure S6).

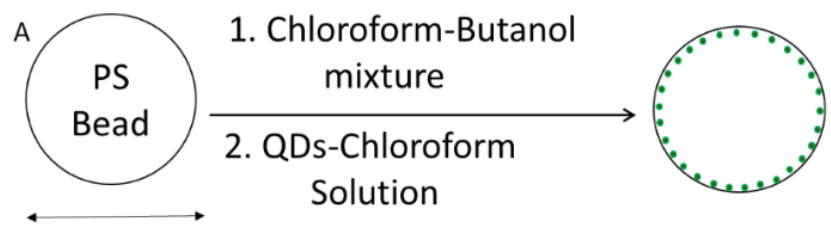

$10 \mu \mathrm{m}$
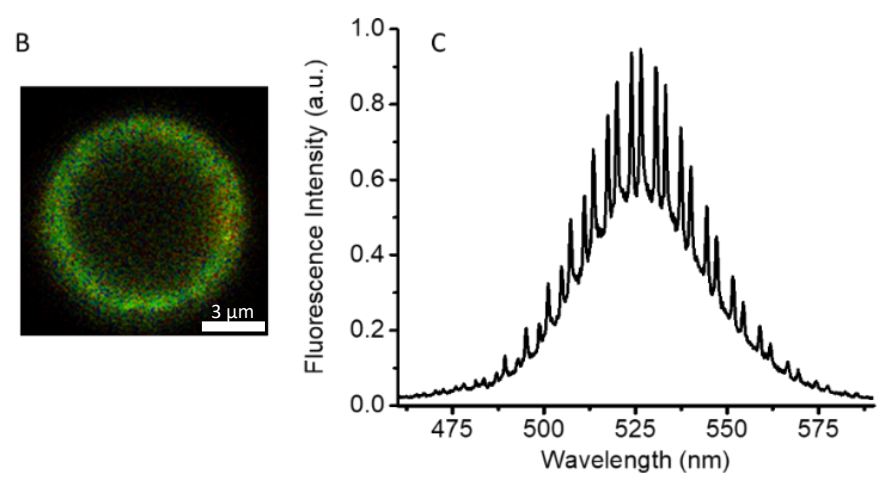

Figure 2: A: Protocol for loading the microspheres with QDs; B: Confocal fluorescence microscopy image of a microsphere loaded with QD 525. C: Emission spectrum of a QD 525 loaded microsphere acquired from a point at the equator.

In a next step, we then used dye nanoparticles (dyeNPs) as acceptors of the WGMs. The dyeNPs were synthesized through nanoprecipitation of poly(methylmethacrylate-co-methacrylic acid) (PMMAMA) together with the salt of a rhodamine B octadecyl ester (R18) with a bulky hydrophobic counterion, tetrakis(pentafluorophenyl)borate (30 wt\% loading relative to the polymer). ${ }^{44,45}$ The resulting dyeNPs have a hydrodynamic diameter of $60 \pm 4 \mathrm{~nm}$ (PDI 0.11), as measured by dynamic light scattering (DLS). The dyeNPs incorporate about 3000 dye molecules per particle (based on their core size of about 35 $\mathrm{nm}$, as determined previously by TEM, and their loading). ${ }^{44}$ This results in a per particle absorbance of about $3.7 \times 10^{8} \mathrm{M}^{-1} \cdot \mathrm{cm}^{-1}$. The use of the hydrophobic, bulky counterion prevents aggregation of the dyes, as indicated by the absorption spectrum approaching that of the dye in organic solution, though a red-shift of the emission spectrum was observed (Figure S7). The insulation of the dyes through the counterions allows maintaining a high quantum yield (QY) even at very high loadings, ${ }^{44,45,46}$ which was determined here to be $36 \pm 5 \%$, resulting in a per particle brightness of $1.3 \times 10^{8} \mathrm{M}^{-1} \cdot \mathrm{cm}^{-1}$. Such dyeNPs have typically shown an efficient and fast energy transfer within the encapsulated dyes inside the dyeNP. ${ }^{18}$ QD-loaded microspheres were then coated with polyethyeneimine (PEI, MW $=1800 \mathrm{~g} / \mathrm{mol}$ ) to provide a positively charged surface. Mixing these microspheres with the negatively charged dyeNPs enables adsorption of the smaller dyeNPs onto the microspheres. In order to probe the energy transfer between QD-excited WGM donors and dyeNP acceptors, we prepared three different batches of microspheres, labeled only with QDs, only with dyeNPs, or with both QDs and dyeNPs. Figure 3 
shows fluorescence microscopy images of these microspheres acquired with filter settings chosen to selectively excite and detect QDs (donor channel, left column), excite and detect dyeNPs (acceptor direct excitation channel, middle column), or excite QDs and detect emission from dyeNPs (energy transfer channel, right column). The observation of microspheres labeled with only QDs or only dyeNPs (Figure 3, upper two rows) shows minimal contributions from emission cross-talks and dyeNPs direct excitation. Finally, acquisition of images from microspheres labeled with both QDs and dyeNPs (Figure 3, bottom row) shows a strong emission in the energy transfer channel. Direct excitation of dyeNPs (panel 8) is minimal compared to the sensitized emission (panel 9). This confirms that the observed emission from dyeNPs in the QD+dyeNPs microspheres indeed originate from energy transfer from QDs, not from direct dye excitation.



Figure 3: Fluorescence microscopy images of microspheres loaded with QD only (top row), adsorbed dyeNP only (middle row) and both QD and dyeNP (bottom row). The images in the first column $(1,4,7)$ were acquired by exciting and detecting the QD fluorescence, whereas images in the $2^{\text {nd }}$ column $(2,5,8)$ were acquired by exciting and detecting the dyeNP fluorescence and images in the $3^{\text {rd }}$ column $(3,6,9)$ were acquired by exciting the QD and detecting fluorescence from the dyeNP. (The insets in panels 5,6 shows a bead coated with 10 times more dyeNP for clarity.) Scale bar: $10 \mu \mathrm{m}$.

Figure 4A shows a typical emission spectrum acquired on a confocal microscope at a point located at the equator of the microsphere. In addition to the QD emission, the spectrum now shows a clear optical signature of dyeNP emission (550-625 nm), despite the fact that the laser excitation at $405 \mathrm{~nm}$ lies far from the dyeNP excitation spectrum. WGM peaks are also clearly visible in the dyeNP emission, 
which shows that part of the dyeNP emission also couples efficiently to redder WGMs. Using Eq. 1 to fit the wavelengths of the modes in the whole spectrum shows that they indeed originate from the same set of WGMs, with radii and microsphere indices of refraction in a comparable range as the QDonly microspheres (Figure S4,B).
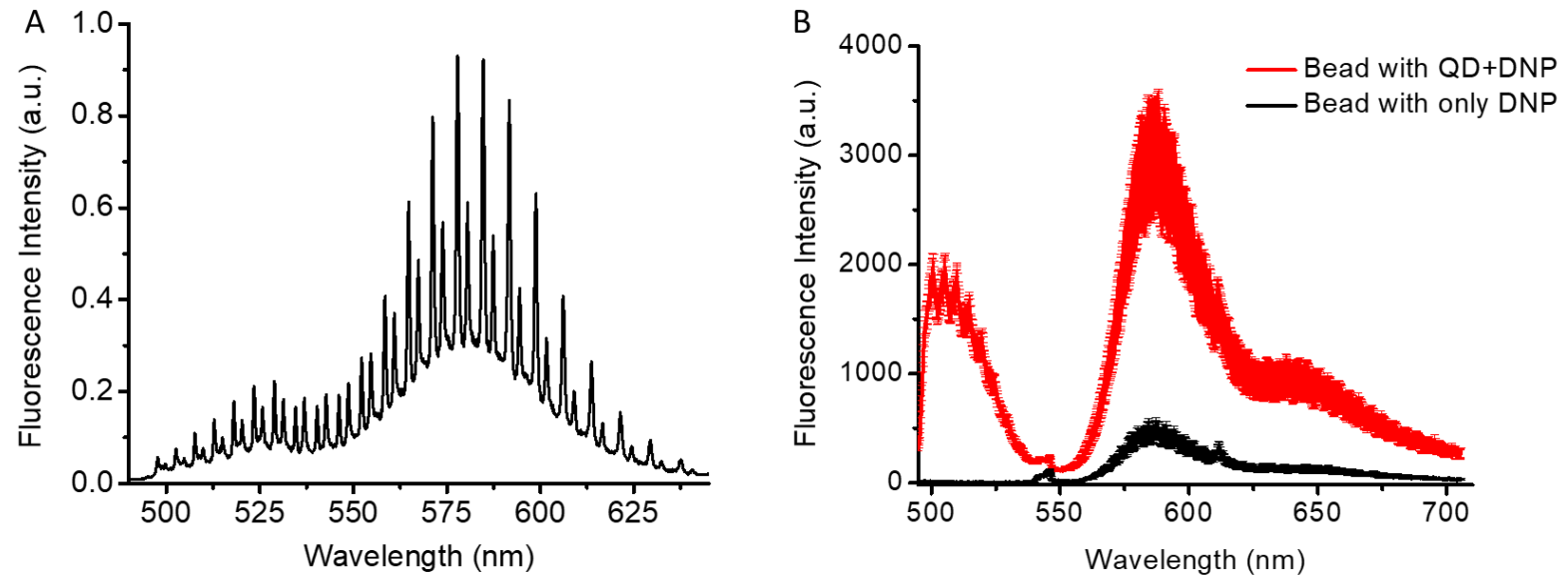

Figure 4: A: Emission spectrum of a QD 525 loaded microsphere with dye NPs adsorbed on its surface, collected by confocal fluorescence microscopy at the equator. B: Average fluorescence spectra of microspheres with and without QDs (QD 505) but with same amount of adsorbed dyeNPs.

To verify that the dyeNP emission mainly originate from energy transfer and not direct excitation, we compared the fluorescence intensity of individual beads with and without QDs coated with the same amount of dyeNPs and illuminated by the same laser intensity. Despite some variation in dyeNP adsorption from one bead to another, Figure 3B clearly shows that the dyeNP fluorescence intensity is much higher in presence of QD donors than in absence of QDs, suggesting that dyeNP emission indeed originate from energy transfer.

We now set out to analyze in more detail the energy transfer rates and efficiencies. The rate of energy transfer, $\Gamma_{\mathrm{ET}}$, from the WGM at wavelength $\lambda$ to an acceptor located at a distance $z$ from the surface of the microsphere can be estimated as:

$$
\Gamma_{E T}(\lambda)=\sigma_{A}(\lambda) \times \frac{c}{n_{s}} \times \frac{\varrho_{S} \times \delta \theta \times 2 \pi R^{2}}{V_{\operatorname{mode}}} \times \frac{I(z)}{I_{\max }}
$$

where $\sigma_{A}(\lambda)$ is the acceptor absorption cross section, $c$ is the speed of light, $\rho_{s}$ is the acceptor density at the surface of the microsphere, $\delta \theta$ is the angular width of the mode at the surface. The third factor of the right-side term in Eq. 2 therefore represents the effective concentration of acceptor species in 
interaction with the mode. $I(z) / I_{\max }$ is the ratio of the WGM intensity at a distance $z$ from the surface of the microsphere to the mode maximum.

Intuitively, the quality factor of a WGM relates to the time that a photon remains confined in this mode before exiting it by scattering or absorption. Thus, when the quality factor is high, a photon circulating in a WGM with a dyeNP adsorbed on its surface comes back many times in contact with this acceptor, increasing the probability of interaction. Indeed, the energy transfer efficiency can be expressed as a function of $\Gamma_{\mathrm{ET}}$, the energy transfer rate from Eq (2) and $\Gamma_{0}$, the rate of WGM relaxation due to all other scattering or absorption processes:

$$
E_{E T}=\frac{\Gamma_{E T}}{\Gamma_{E T}+\Gamma_{0}} .
$$

Since the Purcell factor of the WGM cavity, and the efficiency of the coupling of the QD emission to the WGM is proportional to the quality factor $Q$, the emission spectrum of a microsphere provides a wealth of information about energy transfer from WGMs to dyeNPs. Indeed, considering $\operatorname{IWGM}_{\mathrm{W}}(\lambda)$ as the intensity of a WGM peak (blue points in Figure $5 A$ ) at wavelength $\lambda$ and $I_{\text {Env }}(\lambda)$ as the envelope intensity at the same wavelength, derived by interpolating the spectrum local minima (red points in Figure 5B) we can estimate the variation of the quality factor at different wavelengths using

$$
Q(\lambda) \propto \frac{I_{W G M}}{I_{E n v}}-1 .
$$
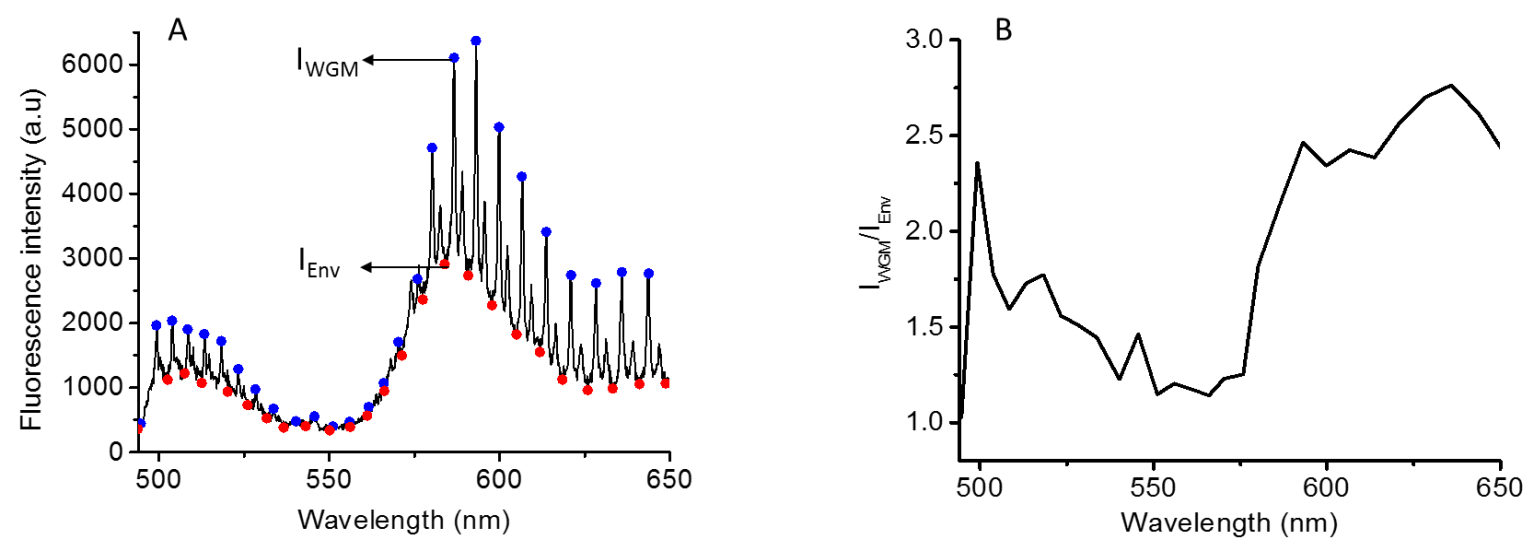

Figure 5: A: Typical confocal emission spectrum collected from a QD 505 labeled microsphere with dye NPs assembled on its surface. $I_{W G M}(\lambda)$ and $I_{\text {Env }}(\lambda)$ respectively denote the intensities of the WGM peak and the envelope at a particular emission wavelength. B: The ratio of peak to envelope intensities vs. the emission wavelength for the spectrum shown in Figure 5A.

Figure 5B shows the evolution of the quality factor for the spectrum shown in Figure 5A. Qualitatively, one can see that the quality factor reaches a minimum around 550-570 nm, where the absorption of 
the dyeNP is maximal, and is maximal in regions of minimal dyeNP absorption, as expected from Eq.2. More quantitatively, the quality factor is inversely proportional to the rates of relaxation of the cavity mode, including contribution from scattering, re-absorption from QDs and absorption from dyeNPs. Considering that the quality factor of QD-only beads is homogeneous across the spectrum, we assume that the rate of WGM relaxation due all scattering and absorption processes other than energy transfer to dyeNP, $\Gamma_{0}$, is independent of wavelength within our limited spectral range. It can thus be inferred from values of $Q$ observed outside of the dyeNP absorption range, at wavelengths longer than $600 \mathrm{~nm}$. The variations of the energy transfer rate with the wavelength, $\Gamma_{\mathrm{ET}}(\lambda)$ can thus be estimated from $Q(\lambda)$, as:

$$
\Gamma_{E T}(\lambda) \propto \frac{1}{Q(\lambda)}-\frac{1}{Q(600 \mathrm{~nm})}
$$
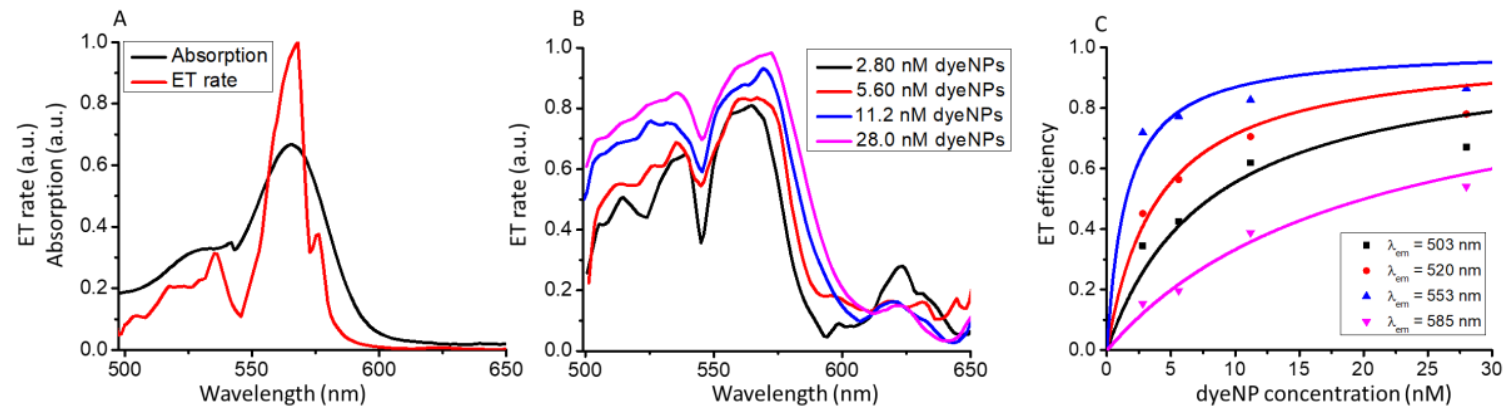

Figure 6. A: Absorption of the dye NPs (black) and energy transfer rate from WGM to dyeNPs in microspheres loaded with QD 505 (red). B: Energy transfer efficiencies as a function of WGM wavelength for different samples of microspheres that have same amount of QD 505 emitters but different amounts of dye NPs. C: Energy transfer efficiency from WGMs at different wavelengths as a function of the concentration of dyeNP acceptors.

As shown in Figure 6A, these variations follow the same shape as the dyeNP absorption spectrum, as expected from Eq. 2. This behavior is also confirmed when the experiment is repeated with QD525 (see Figure S8). Analyzing the spectra obtained with the high-resolution fluorimeter, the variation of $Q$ (either from TE or TM modes) with the wavelength is observed which showed a clear minimum corresponding to the absorption maximum of the dyeNPs (Figure S9). It confirms that the gallery modes at the range where the dyeNPs absorbs the highest are the most affected due to the energy transfer. Similarly, the energy transfer efficiency can be estimated from Eq. 4. Figure 6B shows the evolution of the average energy transfer efficiencies obtained from populations of microspheres mixed with different concentrations of dyeNPs. Similarly to the rate in Figure $6 \mathrm{~A}$, the transfer efficiency is maximal at the peak of dyeNP absorption. In addition, when the quantity of adsorbed 
dyeNP acceptor increases, the energy transfer efficiency increases. As shown in Figure 6C, at each wavelength, $E(\lambda)$ varies with the dyeNP acceptor concentration, $\rho_{\mathrm{s}}$, as expected from Eq. 2 and 3, which can be rewritten as:

$$
E(\lambda)=\frac{\rho_{S}}{\rho_{S}+\rho_{0}(\lambda)}
$$

where $\rho_{0}(\lambda)$ is a constant corresponding to the concentration of acceptors needed to reach $E(\lambda)=50 \%$ at this specific wavelength.
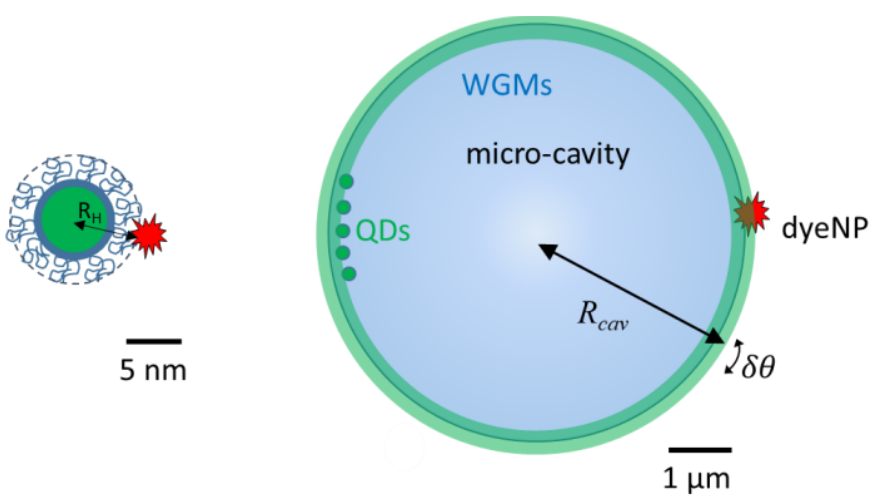

\begin{tabular}{l|c|c} 
& $\begin{array}{c}\text { FRET } \\
\text { Single QD, 1 acceptor/QD }\end{array}$ & $\begin{array}{c}\text { WGET } \\
\text { Collective energy transfer }\end{array}$ \\
\hline Donor surface & $4 \pi \times \mathrm{R}_{\mathrm{H}}{ }^{2} \approx 400 \mathrm{~nm}^{2}$ & $\begin{array}{c}\delta \theta \times 2 \pi R_{\text {cav }}^{2} \approx 4 \times 10^{7} \mathrm{~nm}^{2} \\
\left(R c_{a v}=5 \mu m ; \delta \theta \sim 0.27\right)\end{array}$ \\
\hline Acceptor density & $1 / 400 \mathrm{~nm}^{-2}$ & $1 / 400 \mathrm{~nm}^{-2}$ \\
\hline \#acceptors/donor & 1 & $\approx 10^{5}$
\end{tabular}

Scheme 2. Comparison between FRET and WGET
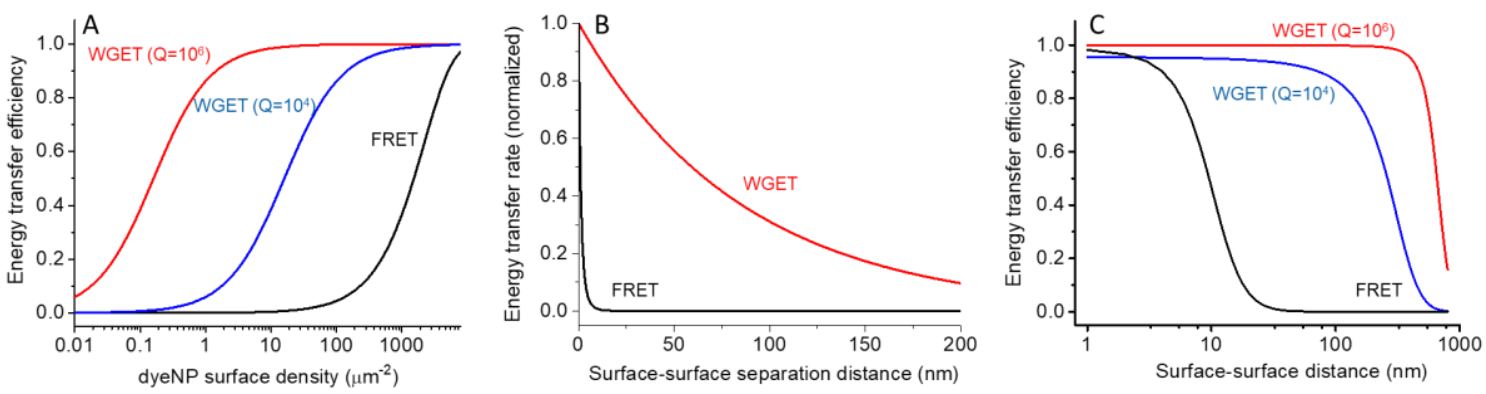

Figure 7. A: Theoretical energy transfer efficiency as a function of dyeNP acceptor surface density for FRET, WGET (quality factors of $10^{4}$ and $10^{6}$ ), with the dyeNP placed directly at the surface. B: Normalized energy transfer rate for FRET and WGET as a function of the surface-to-surface separation distance. C: Energy transfer efficiency as a function of the surface-to-surface separation distance for FRET and WGET (quality factors of $10^{4}$ and $10^{6}$ ), with a dyeNP density $=1 / 400 \mathrm{~nm}^{2}$ (corresponding to 1 dyeNP/QD in the case of FRET). 
In this final section, we would like to discuss the specificities of energy transfer from WGM donors to dyeNP acceptors (WGET) that are expected to make them particularly efficient compared to FRET. For this, we evaluate our system from a theoretical point of view to obtain an estimation of its possibilities: We first note that, in the case of WGET, the donor is a WGM mode, which is excited by many QDs, which provides a considerable brightness when compared with single donors. Then, as an illustration, we would like to compare energy transfer to dyeNPs from WGM donors (WGET) and from isolated QDs (FRET). In the context of biosensing, where for example capture antibodies would be used to bring dyeNP acceptors in close proximity of FRET or WGET donors, we compare the energy transfer efficiencies in conditions where the same amount of capture antibody is used, or equivalently, with the same surface density of dyeNP acceptors (see Scheme 2). In the case of FRET, dyeNPs present very large absorption cross-sections, and we consider the extreme case where QDs undergo a FRET efficiency of 1 as soon as they are conjugated to at least one acceptor. Taking into account the surface chemistry of the QDs and a typical hydrodynamic radius $R_{H}$ of $6 \mathrm{~nm},{ }^{47}$ the total FRET efficiency can be expressed as a function of $\rho_{\mathrm{s}}$, the acceptor surface density, as $E_{F R E T}(\lambda)=\left(1-e^{-\rho_{S^{*}} 4 \pi R_{H}^{2}}\right)$, corresponding to the fraction of QDs conjugated to at least one acceptor, assuming a Poisson distribution of the acceptor:QD ratio. ${ }^{48}$ Figure 7A shows the predicted energy transfer efficiency for FRET and WGET as a function of the acceptor surface density, and for different quality factor $Q$, when dyeNP are directly adsorbed at the FRET or WGET donor surface. We take into account the fact that the dyeNPs, due to their large size, cannot be approximated by a single dipole, and we integrate FRET and WGET transfer rates over the volume of the dyeNP. The strikingly superior efficiency of WGET originates from the delocalized and collective nature of the WGM: instead of being confined to $4 \pi R_{H}^{2} \sim 400 \mathrm{~nm}^{2}$ as in the case of FRET, the WGM is delocalized over $\delta \theta \times 2 \pi R^{2} \sim 42 \mu \mathrm{m}^{2}$, an effective surface that is $10^{5}$ larger. In addition, the high quality factor enables multiple interactions between the WGM field and each acceptor. This largely compensates for the lower electromagnetic intensity at the surface of the microsphere, compared to the higher near-field intensity in the case of FRET.

When the dyeNP acceptor is located farther away from the surface, as would be expected in a biosensing scheme, the acceptor experiences a donor intensity that decays as a function of the separation between the donor surface and the acceptor surface, $d$. In the case of FRET, the donor intensity varies as $\mathrm{d}^{-6}$. In contrast, in the case of WGET, the intensity decay is well approximated by a mono-exponential with a characteristic length of $85 \mathrm{~nm}$ for the microspheres used in this work (this penetration depth into the external medium varies slightly with the wavelength and the microsphere radius, see Figure S10). As shown in Figure 7B, the donor intensity, and thus the energy transfer rate extends much farther away from the surface for WGET than for FRET. To compare how energy transfer 
efficiency varies with separation distance in FRET and WGET, we assume a surface density of 1 acceptor dyeNP for $400 \mathrm{~nm}^{2}$ - in the case of FRET this corresponds to a 1:1 dyeNP:QD ratio. As shown in Figure 7C, FRET efficiency reaches 50\% at a QD surface-to-dyeNP surface of $\sim 10 \mathrm{~nm}$. In comparison, WGET is efficient over larger distances: when $Q=10^{4}$, the $50 \%$ distance is $\sim 47 \mathrm{~nm}$, while it reaches $\sim 100 \mathrm{~nm}$ when $\mathrm{Q}=10^{6}$. This makes WGET advantageous to probe large biomolecular assemblies such as antibody-antigen sandwiches, large oligonucleotides, or even small viruses.

\section{Conclusion:}

In summary, we explored the use of spherical micro-resonators to perform energy transfer from the cavity resonance modes (WGM) to outside optical acceptors, in the context of optical sensing. We fabricated polystyrene micro-cavities where bright and photostable colloidal quantum dots are placed inside to act as optical donors. Spectroscopic characterization revealed the efficient coupling of the quantum dot emission to the modes of the microcavity with high $(>4000)$ quality factors. Bright and highly absorbing dye NPs were assembled on the micro-cavities to act as optical acceptors. Analysis of the emission spectrum from the cavity enabled us to determine the nature of the energy transfer processes from WGM to these acceptors both qualitatively and quantitatively, as a function of the spectral overlap and the density of acceptors. Our experimental data and modeling show that energy transfer from WGM to acceptors placed in the vicinity of the cavity surface is greatly enhanced by the quality factor of the cavity. The energy transfer can be efficient over distances larger than $100 \mathrm{~nm}$, compared to typically $10 \mathrm{~nm}$ for FRET. This would enable the use of WGET to design highly sensitive homogenous assays to detect large biomolecular entities from peptides, proteins or oligonucleotides to large antibody-antigen sandwiches or small viruses. Bio-specificity could be easily imparted on these microspheres by conjugating whole antibodies or other capture probes without the size constraints encountered in FRET assays. Further developments of WGET could thus enable faster, user friendly and more sensitive tools for bio-detection.

\section{Experimental section:}

Materials: Polystyrene microspheres (10 $\mu \mathrm{m}$ in diameter), poly(methyl methacrylate-co-methacrylic acid) (PMMAMA noted here as PMMA, 1,3\% methacrylic acid, $M_{n} \sim 15,000, M_{w} \sim 34,000$, ) and all other chemicals were purchased from Sigma-Aldrich (Merck).

Synthesis of QDs: Three different CdSe-based core/shell QD samples with emission maxima wavelengths at $505 \mathrm{~nm}$ (QD505) and $525 \mathrm{~nm}$ (QD525) were synthesized using adapted protocols from previously described methods (see SI for synthesis details). 
Loading of QDs into polystyrene microspheres: QDs were precipitated from hexane using ethanol and resuspended in chloroform at a concentration of $15 \mu \mathrm{M} . \quad 5 \mu \mathrm{L}\left(\sim 10^{6}\right.$ beads) aqueous solution of polystyrene microspheres were added to $2 \mathrm{~mL}$ of ethanol and centrifuged at $2000 \mathrm{~g}$ for 5 seconds. The supernatant was removed leaving the solid microspheres at the bottom. The beads were resuspended using $1 \mathrm{~mL}$ of butanol:chloroform mixture $(3: 7,2: 1$ in volume, respectively) and $25 \mu \mathrm{L}$ of the QD solution (QD505, QD525, respectively) were added, for a $0.375 \mu \mathrm{M}$ final concentration. The solutions were mixed on a rotary agitator for $4 \mathrm{~h}$ at room temperature. The beads were then centrifuged at 2000 $\mathrm{g}$ for 10 seconds and washed with ethanol 3 times to remove solvent and excess QDs. The beads were finally resuspended in $500 \mu \mathrm{L}$ of water.

Synthesis of dye nanoparticles: R18/F5-TPB, the salt of Rhodamine B octadecyl ester with tetrakis(pentafluorophenyl)borate, was synthesized through dye exchange followed by purification through column chromatography as described previously. ${ }^{44,45} \mathrm{~A}$ solution of PMMAMA in acetonitrile ( $2 \mathrm{mg} \mathrm{mL}^{-1}$ ) containing $30 \mathrm{wt} \%$ of R18/F5-TPB (relative to the polymer) was added quickly and under stirring (shaking) using a micropipette to a 10-fold volume excess of $20 \mathrm{mM}$ phosphate buffer at $\mathrm{pH}$ 7.4, followed by a five-fold dilution.

Deposition of dye nanoparticles on the QD filled PS beads: The QD-loaded beads was incubated for $2 \mathrm{~h}$ in an aqueous solution of polyethylenimine $\left(\mathrm{PEI}, \mathrm{M}_{\mathrm{w}}=1800 \mathrm{~g} / \mathrm{mol}, 10 \mu \mathrm{g} / \mathrm{mL}\right.$ ). Then $1.5 \mathrm{~mL}$ ethanol was added and the beads were centrifuged at $3000 \mathrm{~g}$ for 5 minutes to remove excess PEI. The beads were resuspended in $500 \mu \mathrm{L}$ of water and the desired volume of dye nanoparticles solution (typically $50 \mu \mathrm{L}$ at $0.04 \mathrm{~g} / \mathrm{L}$ ) was added and mixed for $2 \mathrm{~h}$. The beads were separated from the unbound dye NPs by centrifuging at $2000 \mathrm{~g}$ for $5 \mathrm{~s}$.

Instrumentation: Absorption spectra were acquired on a UV-1800 Shimadzu spectrometer. Solution phase fluorescence spectra were acquired with F900 spectrometer (Edinburgh Instruments). Electron microscopy was performed on a Jeol 2010F microscope. Fluorescence micro-spectroscopy was performed on a confocal microscope set up (Microtime 200, Picoquant), using a $405 \mathrm{~nm}$ excitation and an attached fluorescence spectrometer (Andor Shamrock 750, 149 gratings/mm) equipped with an Andor Newton CCD sensor.

Wide-field FRET fluorescence microscopy was performed on a Olympus IX-71 microscope equipped with a 100x NA 1.45 objective, a Quantem 512SC CCD camera and three filter cubes: QD $\rightarrow Q D$ (exc:425-60, dichroic: LP565 , em: 605-40), dye $\rightarrow$ dye direct excitation: (exc: 560-55, dichroic: LP595 , em: 645-75) and QD $\rightarrow$ dye (exc:435-40/25, dichroic: LP565 , em: LP590 ). 
Acknowledgements. This project has received funding from the European Union's Horizon 2020 research and innovation program under the Marie Sklodowska-Curie grant agreement No. 754387. The authors would like to thank JC Weeber (ICB, Université de Bourgogne) for providing support for high resolution fluorescence spectroscopy.

Supporting Information Available: The Supporting Information is available free of charge on the ACS Publications website. QD synthesis protocol, additional spectroscopic characterization, dye NP structure.

\section{References:}

(1) Takkinen, K.; Žvirblienè, A. Recent Advances in Homogenous Immunoassays Based on Resonance Energy Transfer. Curr. Opin. Biotechnol. 2019, 55, 16-22.

(2) Algar, W. R.; Hildebrandt, N.; Vogel, S. S.; Medintz, I. L. FRET as a Biomolecular Research Tool - Understanding Its Potential While Avoiding Pitfalls. Nat. Methods 2019, 16, 815-829.

(3) Sapsford, K. E.; Berti, L.; Medintz, I. L. Materials for Fluorescence Resonance Energy Transfer Analysis: Beyond Traditional Donor-Acceptor Combinations. Angew. Chem. Int. Ed. 2006, 45, 4562-4589.

(4) Hemerka, J. N.; Wang, D.; Weng, Y.; Lu, W.; Kaushik, R. S.; Jin, J.; Harmon, A. F.; Li, F. Detection and Characterization of Influenza A Virus PA-PB2 Interaction through a Bimolecular Fluorescence Complementation Assay. J. Virol. 2009, 83, 3944-3955.

(5) Kodama, Y.; Wada, M. Simultaneous Visualization of Two Protein Complexes in a Single Plant Cell Using Multicolor Fluorescence Complementation Analysis. Plant Mol. Biol. 2009, 70, 211.

(6) Hieb, A. R.; Halsey, W. A.; Betterton, M. D.; Perkins, T. T.; Kugel, J. F.; Goodrich, J. A. TFIIA Changes the Conformation of the DNA in TBP/TATA Complexes and Increases Their Kinetic Stability. J. Mol. Biol. 2007, 372, 619-632.

(7) Dragan, A. I.; Klass, J.; Read, C.; Churchill, M. E. A.; Crane-Robinson, C.; Privalov, P. L. DNA Binding of a Non-Sequence-Specific HMG-D Protein Is Entropy Driven with a Substantial NonElectrostatic Contribution. J. Mol. Biol. 2003, 331, 795-813.

(8) Rehman, S.; Gladman, J. T.; Periasamy, A.; Sun, Y.; Mahadevan, M. S. Development of an APFRET Based Analysis for Characterizing RNA-Protein Interactions in Myotonic Dystrophy (DM1). PLOS ONE 2014, 9, e95957.

(9) Shih, W. M.; Gryczynski, Z.; Lakowicz, J. R.; Spudich, J. A. A FRET-Based Sensor Reveals Large ATP Hydrolysis-Induced Conformational Changes and Three Distinct States of the Molecular Motor Myosin. Cell 2000, 102, 683-694.

(10) Mekler, V.; Kortkhonjia, E.; Mukhopadhyay, J.; Knight, J.; Revyakin, A.; Kapanidis, A. N.; Niu, W.; Ebright, Y. W.; Levy, R.; Ebright, R. H. Structural Organization of Bacterial RNA Polymerase Holoenzyme and the RNA Polymerase-Promoter Open Complex. Cell 2002, 108, 599-614.

(11) Xu, S.; Xu, S.; Zhu, Y.; Xu, W.; Zhou, P.; Zhou, C.; Dong, B.; Song, H. A Novel Upconversion, Fluorescence Resonance Energy Transfer Biosensor (FRET) for Sensitive Detection of Lead Ions in Human Serum. Nanoscale 2014, 6, 12573-12579.

(12) Ma, C.; Zeng, F.; Huang, L.; Wu, S. FRET-Based Ratiometric Detection System for Mercury lons in Water with Polymeric Particles as Scaffolds. J. Phys. Chem. B 2011, 115, 874-882.

(13) Pulli, T.; Höyhtyä, M.; Söderlund, H.; Takkinen, K. One-Step Homogeneous Immunoassay for Small Analytes. Anal. Chem. 2005, 77, 2637-2642.

(14) Niemi, M. H.; Turunen, L.; Pulli, T.; Nevanen, T. K.; Höyhtyä, M.; Söderlund, H.; Rouvinen, J.; Takkinen, K. A Structural Insight into the Molecular Recognition of a (-)-Delta9Tetrahydrocannabinol and the Development of a Sensitive, One-Step, Homogeneous Immunocomplex-Based Assay for Its Detection. J. Mol. Biol. 2010, 400, 803-814. 
(15) Takahashi, T.; Mihara, H. FRET Detection of Amyloid $\beta$-Peptide Oligomerization Using a Fluorescent Protein Probe Presenting a Pseudo-Amyloid Structure. Chem. Commun. 2012, 48, 1568-1570.

(16) Aliyu, S. H.; Aliyu, M. H.; Salihu, H. M.; Parmar, S.; Jalal, H.; Curran, M. D. Rapid Detection and Quantitation of Hepatitis B Virus DNA by Real-Time PCR Using a New Fluorescent (FRET) Detection System. J. Clin. Virol. 2004, 30, 191-195.

(17) Deng, R.; Wang, J.; Chen, R.; Huang, W.; Liu, X. Enabling Förster Resonance Energy Transfer from Large Nanocrystals through Energy Migration. J. Am. Chem. Soc. 2016, 138, 1597215979.

(18) Trofymchuk, K.; Reisch, A.; Didier, P.; Fras, F.; Gilliot, P.; Mely, Y.; Klymchenko, A. S. Giant Light-Harvesting Nanoantenna for Single-Molecule Detection in Ambient Light. Nat. Photonics 2017, 11, 657-663.

(19) Yun, C. S.; Javier, A.; Jennings, T.; Fisher, M.; Hira, S.; Peterson, S.; Hopkins, B.; Reich, N. O.; Strouse, G. F. Nanometal Surface Energy Transfer in Optical Rulers, Breaking the FRET Barrier. J. Am. Chem. Soc. 2005, 127, 3115-3119.

(20) Pons, T.; Medintz, I. L.; Sapsford, K. E.; Higashiya, S.; Grimes, A. F.; English, D. S.; Mattoussi, H. On the Quenching of Semiconductor Quantum Dot Photoluminescence by Proximal Gold Nanoparticles. Nano Lett. 2007, 7, 3157-3164.

(21) Chen, C.; Hildebrandt, N. Resonance Energy Transfer to Gold Nanoparticles: NSET Defeats FRET. TrAC Trends Anal. Chem. 2020, 123, 115748.

(22) Algar, W. R.; Kim, H.; Medintz, I. L.; Hildebrandt, N. Emerging Non-Traditional Förster Resonance Energy Transfer Configurations with Semiconductor Quantum Dots: Investigations and Applications. Coord. Chem. Rev. 2014, 263-264, 65-85.

(23) Hildebrandt, N.; Spillmann, C. M.; Algar, W. R.; Pons, T.; Stewart, M. H.; Oh, E.; Susumu, K.; Díaz, S. A.; Delehanty, J. B.; Medintz, I. L. Energy Transfer with Semiconductor Quantum Dot Bioconjugates: A Versatile Platform for Biosensing, Energy Harvesting, and Other Developing Applications. Chem. Rev. 2017, 117, 536-711.

(24) Humar, M.; Gather, M. C.; Yun, S.-H. Cellular Dye Lasers: Lasing Thresholds and Sensing in a Planar Resonator. Opt. Express 2015, 23, 27865-27879.

(25) Chen, Y.-C.; Tan, X.; Sun, Q.; Chen, Q.; Wang, W.; Fan, X. Laser-Emission Imaging of Nuclear Biomarkers for High-Contrast Cancer Screening and Immunodiagnosis. Nat. Biomed. Eng. 2017, 1, 724-735.

(26) Vollmer, F.; Arnold, S. Whispering-Gallery-Mode Biosensing: Label-Free Detection down to Single Molecules. Nat. Methods 2008, 5, 591-596.

(27) Baaske, M. D.; Foreman, M. R.; Vollmer, F. Single-Molecule Nucleic Acid Interactions Monitored on a Label-Free Microcavity Biosensor Platform. Nat. Nanotechnol. 2014, 9, 933939.

(28) Zhang, W.; Ganesh, N.; Block, I. D.; Cunningham, B. T. High Sensitivity Photonic Crystal Biosensor Incorporating Nanorod Structures for Enhanced Surface Area. Sens. Actuators $B$ Chem. 2008, 131, 279-284.

(29) Guo, Y.; Ye, J. Y.; Divin, C.; Huang, B.; Thomas, T. P.; Baker, Jr., James R.; Norris, T. B. RealTime Biomolecular Binding Detection Using a Sensitive Photonic Crystal Biosensor. Anal. Chem. 2010, 82, 5211-5218.

(30) Gohring, J. T.; Dale, P. S.; Fan, X. Detection of HER2 Breast Cancer Biomarker Using the OptoFluidic Ring Resonator Biosensor. Sens. Actuators B Chem. 2010, 146, 226-230.

(31) Flueckiger, J.; Schmidt, S.; Donzella, V.; Sherwali, A.; Ratner, D. M.; Chrostowski, L.; Cheung, K. C. Sub-Wavelength Grating for Enhanced Ring Resonator Biosensor. Opt. Express 2016, 24, 15672-15686.

(32) Andrew, P.; Barnes, W. L. Förster Energy Transfer in an Optical Microcavity. Science 2000, 290, 785-788. 
(33) Folan, L. M.; Arnold, S.; Druger, S. D. Enhanced Energy Transfer within a Microparticle. Chem. Phys. Lett. 1985, 118, 322-327.

(34) Kushida, S.; Braam, D.; Dao, T. D.; Saito, H.; Shibasaki, K.; Ishii, S.; Nagao, T.; Saeki, A.; Kuwabara, J.; Kanbara, T.; Kijima, M.; Lorke, A.; Yamamoto, Y. Conjugated Polymer Blend Microspheres for Efficient, Long-Range Light Energy Transfer. ACS Nano 2016, 10, 5543-5549.

(35) Götzinger, S.; de S. Menezes, L.; Mazzei, A.; Kühn, S.; Sandoghdar, V.; Benson, O. Controlled Photon Transfer between Two Individual Nanoemitters via Shared High-Q Modes of a Microsphere Resonator. Nano Lett. 2006, 6, 1151-1154.

(36) Ahn, W.; Hong, Y.; Boriskina, S. V.; Reinhard, B. M. Demonstration of Efficient On-Chip Photon Transfer in Self-Assembled Optoplasmonic Networks. ACS Nano 2013, 7, 4470-4478.

(37) Clapp, A. R.; Medintz, I. L.; Mauro, J. M.; Fisher, B. R.; Bawendi, M. G.; Mattoussi, H. Fluorescence Resonance Energy Transfer between Quantum Dot Donors and Dye-Labeled Protein Acceptors. J. Am. Chem. Soc. 2004, 126, 301-310.

(38) Reisch, A.; Klymchenko, A. S. Fluorescent Polymer Nanoparticles Based on Dyes: Seeking Brighter Tools for Bioimaging. Small 2016, 12, 1968-1992.

(39) Pang, S.; Beckham, R. E.; Meissner, K. E. Quantum Dot-Embedded Microspheres for Remote Refractive Index Sensing. Appl. Phys. Lett. 2008, 92, 221108.

(40) Lam, C. C.; Leung, P. T.; Young, K. Explicit Asymptotic Formulas for the Positions, Widths, and Strengths of Resonances in Mie Scattering. JOSA B 1992, 9, 1585-1592.

(41) Li, Y.; Abolmaali, F.; Allen, K. W.; Limberopoulos, N. I.; Urbas, A.; Rakovich, Y.; Maslov, A. V.; Astratov, V. N. Whispering Gallery Mode Hybridization in Photonic Molecules. Laser Photonics Rev. 2017, 11, 1600278.

(42) Sultanova, N.; Kasarova, S.; Nikolov, I. Dispersion Properties of Optical Polymers. Acta Phys. Pol. A 2009, 116, 585-587.

(43) Lutti, J.; Langbein, W.; Borri, P. High Q Optical Resonances of Polystyrene Microspheres in Water Controlled by Optical Tweezers. Appl. Phys. Lett. 2007, 91, 141116.

(44) Reisch, A.; Trofymchuk, K.; Runser, A.; Fleith, G.; Rawiso, M.; Klymchenko, A. S. Tailoring Fluorescence Brightness and Switching of Nanoparticles through Dye Organization in the Polymer Matrix. ACS Appl. Mater. Interfaces 2017, 9, 43030-43042.

(45) Reisch, A.; Didier, P.; Richert, L.; Oncul, S.; Arntz, Y.; Mély, Y.; Klymchenko, A. S. Collective Fluorescence Switching of Counterion-Assembled Dyes in Polymer Nanoparticles. Nat. Commun. 2014, 5, 4089.

(46) Andreiuk, B.; Reisch, A.; Bernhardt, E.; Klymchenko, A. S. Fighting Aggregation-Caused Quenching and Leakage of Dyes in Fluorescent Polymer Nanoparticles: Universal Role of Counterion. Chem. - Asian J. 2019, 14, 836-846.

(47) Debayle, M.; Balloul, E.; Dembele, F.; Xu, X.; Hanafi, M.; Ribot, F.; Monzel, C.; Coppey, M.; Fragola, A.; Dahan, M.; Pons, T.; Lequeux, N. Zwitterionic Polymer Ligands: An Ideal Surface Coating to Totally Suppress Protein-Nanoparticle Corona Formation? Biomaterials 2019, 219, 119357.

(48) Pons, T.; Medintz, I. L.; Wang, X.; English, D. S.; Mattoussi, H. Solution-Phase Single Quantum Dot Fluorescence Resonance Energy Transfer. J. Am. Chem. Soc. 2006, 128, 15324-15331. 
$-1 \mathrm{~N}$ 